\title{
A New Process for the Fabrication of SiC Power Devices and Systems on SiCOI (Silicon Carbide On Insulator) Substrates
}

\author{
François Templier, Nicolas Daval ${ }^{1}$, Léa Di Cioccio, Daniel Bourgeat, Fabrice Letertre ${ }^{1}$, \\ Dominique Planson ${ }^{2}$, Jean-Pierre Chante ${ }^{2}$ and Thierry Billon \\ CEA-LETI, 17 rue des Martyrs \\ 38054 Grenoble, France \\ ${ }^{1}$ SOITEC Silicon On Insulator Technologies, Parc Technologique des Fontaines \\ 38190 Bernin, France. \\ ${ }^{2}$ CEGELY, INSA de Lyon, Bat. Léonard de Vinci, 20 avenue Albert Einstein \\ 69621 Villeurbanne, France.
}

\begin{abstract}
Feasibility of 4H-SiC epitaxy on SiCOI substrates has been demonstrated, with high quality of obtained layers. Power Schottky diodes were designed and fabricated on these new structures, and exhibited very interesting electrical performance, particularly in reverse mode, with $\mathrm{V}_{\mathrm{br}} \sim$ $1000 \mathrm{~V}$. This technology is very promising for the realization of monolithic SiC power systems.
\end{abstract}

\section{INTRODUCTION}

After years of promising development, Silicon Carbide power devices are now on the potentially high volume market of discrete components, with the first commercially available Schottky diodes. However, the small size, high cost of SiC substrates are still disadvantages which limit fast expansion of such devices. In another hand, in the field of integrated power systems, SiC hasn't yet emerged. For power systems, SOI (Silicon On Insulator) substrates can be used for the realization of monolithic circuits such as power amplifiers for automotive audio application, with power range up to $\sim 100 \mathrm{~W}$ [1]. Considering the low breakdown field $(0.3 \mathrm{MV} / \mathrm{cm})$ and rather poor thermal conductivity $(1.3 \mathrm{~W} / \mathrm{cm} . \mathrm{K})$ of Silicon, it seems difficult to forecast higher power with this technology.

$\mathrm{SiCOI}$ (Silicon Carbide On Insulator) material, made by the Smart Cut ${ }^{\mathrm{TM}}$ process, is a promising substrate for power applications, providing the excellent breakdown field and thermal conductivity of $4 \mathrm{H}-\mathrm{SiC}$, on 4 -in. or more wafer sizes, which are silicon line compatible. The first demonstration of a SiCOI substrate was made by LETI in 1996 [2]. This first wafer was followed by an active research in order to recover the semiconductor properties of the transferred SiC layer [3]. Nowadays those improvements allow the fabrication of power devices. Recently we presented the first demonstration of power Schottky diodes on 4" SiCOI substrate [4]. The thickness of the $\mathrm{SiC}$ transferred layer is in the order of $0.5 \mu \mathrm{m}$. By designing an original structure involving a sloped Schottky contact, we targeted a $600 \mathrm{~V} / 1$ A rectifier. After full fabrication of the devices on a 4" silicon line, reverse characteristics with $\mathrm{V}_{\mathrm{br}} \sim 300 \mathrm{~V}$ were obtained, while forward current was much lower $\left(\sim 1 / 100^{\text {th }}\right)$ than expected. This low current was interpreted as the result of failure in the ohmic contact process step. This first achievement was interesting, however we were convinced that the limited thickness of the transferred layer is not intrinsically very favourable for high current densities. In this paper we present an important improvement for the SiCOI substrates, which consist of growing epitaxial SiC layers on top of the transferred layers. We will describe these epitaxies, the design and realization of the first demonstrators of 
power Schottky diodes on such structures, which we believe is the first step to high power monolithic $\mathrm{SiC}$ systems.

\section{EPITAXY OF 4H-SiC ON SiCOI SUBSTRATES}

The CVD process developed here is carried out in an home made cold wall reactor described elsewhere [5]. In the present case, we have used SiCOI substrates with poly-SiC handle substrate, instead of silicon. The reactants gases are silane and propane mixed in a pure hydrogen carrier gas. Wafers are placed on a SiC coated-susceptor. Growth is undergone at atmospheric pressure and typical growth temperature is $1450^{\circ} \mathrm{C}$. Growth rate, doping level and morphology of the epitaxy were compared to those obtained on full $4 \mathrm{H}-\mathrm{SiC}$ wafers. Prior to growth a slight $\mathrm{H}_{2}$ etch was performed.

Though the high epitaxy temperature, no oxide degradation was observed. Due to the high SiCOI quality, no oxide $\mathrm{H}_{2}$ etching defects were observed unlike what was previously published for 3C$\mathrm{SiC}$ on SOI $[6,7]$. Only some voids could be seen (Figure 1). Transmission Electron Microscopy of structures after epitaxy is shown in figure 3.

Growth rate, and intentional doping level of epitaxy on SiCOI are similar to those obtained by homoepitaxy on full $4 \mathrm{H} \mathrm{SiC}$ bulk wafers. With respect to these results the following structures were grown (figure 2). These epitaxies were made for the purpose of evaluating power Schottky diodes $\left(3 \mu \mathrm{m} ; \sim 1 \times 10^{16} \mathrm{~cm}^{-3}\right)$. However, it is important to notice that this is a very versatile technology, as any thickness, any doping type or level, and any combination of epitaxial layers can be chosen. Thus, potentially any kind of device can be made using this technology. Substrate diameter: Here we have used 2-in. SiCOI substrate, but one should mention that there is no obstacle to make SiCOI substrates using 4-in. (or more) low cost poly-SiC, which provide the wafers to be processed on standard silicon lines. For $\mathrm{SiC}$ epitaxy, it is now possible to perform it on 4-in. wafer diameter [8].

\section{DESIGN AND REALIZATION OF POWER SCHOTTKY DIODES}

Device structure: Lateral devices were designed [9], giving the schematic structure shown in figure 4. Edge terminations consist of a B-implanted ring around the Schottky contact. Ohmic contact is made on an $\mathrm{N}^{+}$-implanted area. It is important to notice that after etching SiC layers on the edges, the device is perfectly insulated laterally from others. The anode makes a central electrode, and is surrounded by a ring-shaped Cathode. The main dimensions are listed in Table I. This standard diode is rated for $100 \mathrm{~mA}$ in forward bias. Interdigited device with $2 \mathrm{~A}$ current rating were also designed.

Influence of doping of the transferred layer: The $\mathrm{SiC}$ transferred layer can be chosen among either $\mathrm{N}$ doped or semi-insulating. In the case this layer is $\mathrm{N}$ doped at the level of $\sim 5 \times 10^{18} \mathrm{~cm}^{-3}$, it will enhance the current flow in the forward mode, but this conducting layer will be detrimental in the reverse mode because of the shielding of the Resurf effect. By using semiinsulating transferred layer, opposite behaviour will occur with lower On-current and improved reverse mode. As this parameter can have a significant influence on the device characteristics [9], it was evaluated on the devices. Properties of the different transferred layers are described in Table I. 


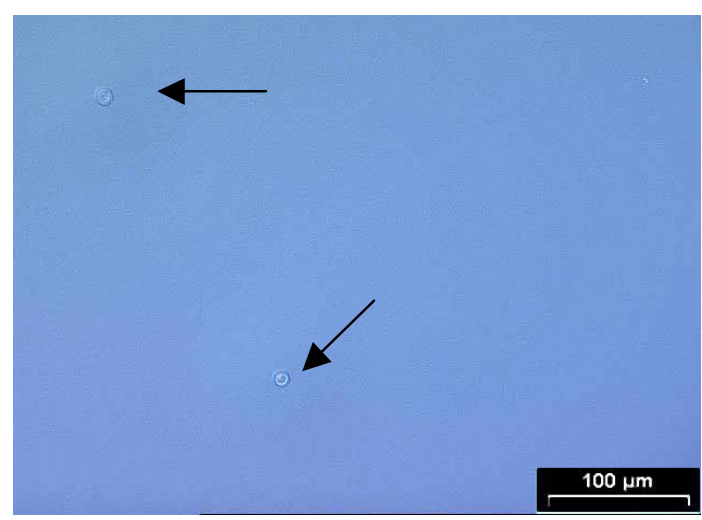

Figure 1. Optical observation of an epitaxy on SiCOI substrate: only small voids can be seen.

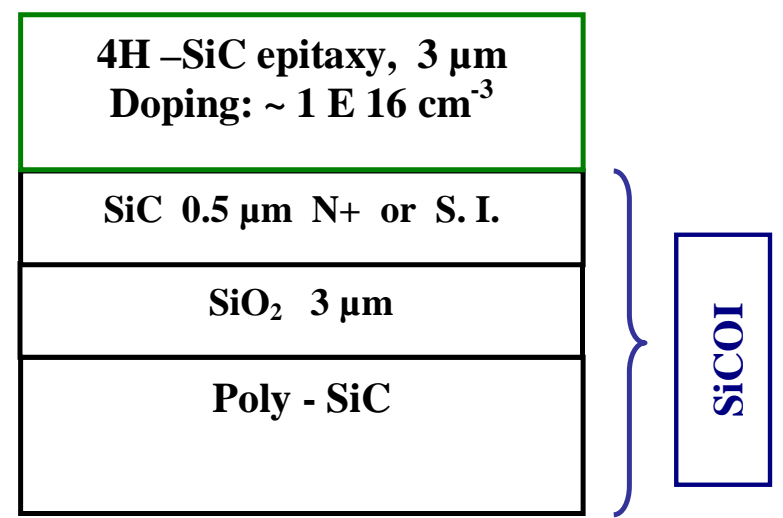

Figure 2. Schematic view of the realized structures (SiCOI substrate + epitaxy).
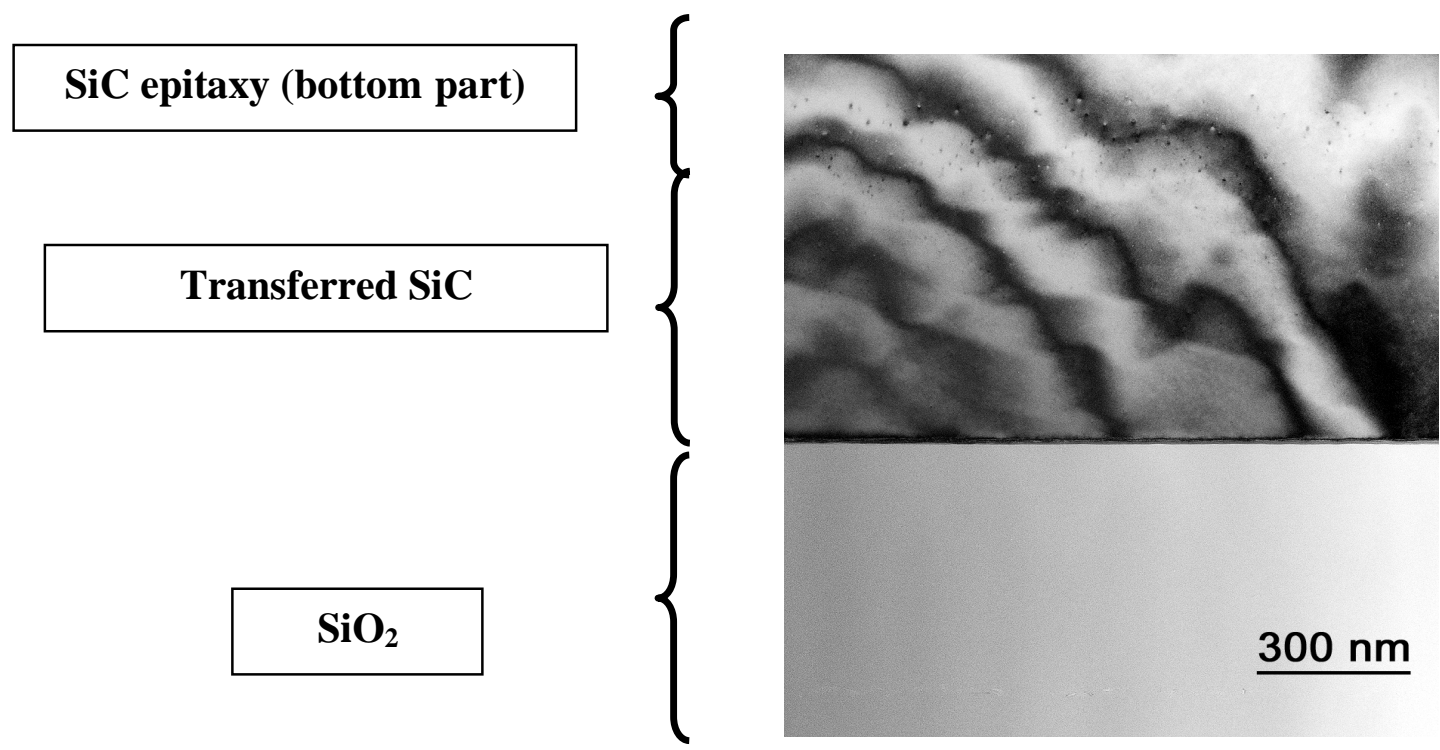

Figure 3. Transmission Electron Microscopy on a SiCOI with lower epitaxy.

B implant. (Edge termination)

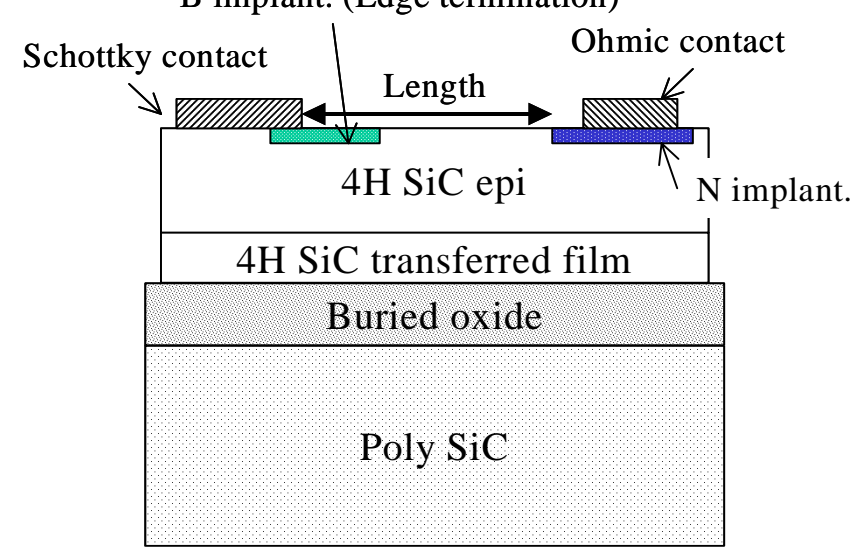

Figure 4. Schematic of the Schottky diodes and substrate structure.

\begin{tabular}{|c|l|}
\hline Buried oxide & \multicolumn{1}{|c|}{$3 \mu \mathrm{m}$} \\
\hline Transferred & 4H SiC, Si face, $0.5 \mu \mathrm{m}$ \\
SiC film & $\begin{array}{l}\text { N+ doped }\left(\sim 5.10^{18} \mathrm{~cm}^{-3}\right) \\
\text { or: Semi-insulating }\end{array}$ \\
\hline Epitaxy & $\begin{array}{l}\text { 4H SiC, } 3 \mu \mathrm{m} \\
\text { N- doped } \sim 1.10^{16} \mathrm{~cm}^{-3}\end{array}$ \\
\hline Diodes: & Length: $21 \mu \mathrm{m}$ \\
Ring shaped: & $\begin{array}{l}\text { Dimension: } 600 \times 200 \mu \mathrm{m} \\
\text { Rating: } 100 \mathrm{~mA}\end{array}$ \\
Interdigited: & $\begin{array}{l}\text { Length: } 21 \mu \mathrm{m} \\
\text { Dimension: } 2000 \times 1250 \mu \mathrm{m} \\
\end{array}$ \\
& Rating: $2 \mathrm{~A}$ \\
\hline
\end{tabular}

Table I. Main characteristics of the structures 
Edge termination: Edge terminations were made using B implantation, with an energy of 30 $\mathrm{keV}$ and a dose of $1 \times 10^{15} \mathrm{~cm}^{-2}$. Annealing was made at $1050^{\circ} \mathrm{C}$. These conditions, which were proposed by Itoh [10], combine rather simple process and good performance. Using this process, the edge termination is effective, by creation of defects [10], and not as a result of p-type-doping, as the annealing temperature $\left(1050^{\circ} \mathrm{C}\right)$ is too low for activating B. However, it is important to mention that the using of SiCOI substrates should be compatible with the realization of "true" ptype edge terminations, as these substrates should withstand a temperature of $1500^{\circ} \mathrm{C}$, which is suitable for p-type annealing temperature for high performance Schottky diodes [11].

\section{Fabrication of the devices:}

The diodes were fabricated using the following process steps:

- Definition of active area: $\mathrm{SiC}$ layers are etched at the edges, which allows perfect galvanic isolation between the devices, thanks to the presence of $\mathrm{SiO}_{2}$ from $\mathrm{SiCOI}$ substrate. The etching is made with ICP high density plasma in an Alcatel 601E tool;

- Passivation: device are passivated with $\mathrm{SiO}_{2}(1.5 \mu \mathrm{m}$ thick) and, at the end of the process, with an organic polyimide-like polymer $(\sim 4 \mu \mathrm{m})$;

- Local Nitrogen doping: in order to provide ohmic contact, nitrogen is locally implanted (box profile, $8 \times 10^{19} \mathrm{~cm}^{-3}$ ), followed by high temperature annealing for dopant activation,

- Local Boron doping: as it was mentioned above, edge terminations are made by local implantation of $\mathrm{B}$ and $1050^{\circ} \mathrm{C}$ annealing,

- Formation of ohmic contacts: Nickel is sputtered, patterned and annealed at $950^{\circ} \mathrm{C}$ for ohmic contact formation. Prior to metallization, surface is cleaned by dipping in HF,

- Formation of Schottky contact: Schottky metallization is made with Ti, which is sputtered, patterned and annealed. Both metallizations are reinforced by $1 \mu \mathrm{m}$ Al layer.

The structure is represented in figure 4. A top view of fully processed wafer is shown in figure 6 .

\section{ELECTRICAL RESULTS:}

Forward bias: Figure 7 shows forward characteristics made on a series of $100 \mathrm{~mA}$-rated diodes, corresponding to structure with conducting transferred layer. Simulated characteristic is also plotted for comparison. The measured current is lower than expected, around one decade below. The behaviour is the same for diodes made on substrate with semi-insulating transferred layer: for the same size, the current rating is $50 \mathrm{~mA}$, and the measured current is around a decade below expected. We suspected a problem in the ohmic contact, and to verify this we analysed ohmic contact pads by SIMS, after removal of Al top metal layer. The result is given in figure 8 . The implanted nitrogen is clearly visible from the $\mathrm{SiC}$ surface, and the $\mathrm{N}$ signal decreases to the level of the epitaxial $\mathrm{SiC}$ drift layer. A significant quantity of oxygen is evidenced at the $\mathrm{Ni} / \mathrm{SiC}$ interface. We could not determine whether this oxygen was from $\mathrm{SiO}_{2}$ phase, or nickel oxide (or other), but its unexpected presence was clearly correlated with the failing ohmic contact. This penalising oxygen contamination was attributed to insufficient cleaning of the $\mathrm{SiC}$ surface prior to Ni metallization, and/or oxygen leak during the ohmic contact annealing at $950^{\circ} \mathrm{C}$.

Contact between Ti and B-implanted SiC: On the wafers we had several TLM test patterns, to evaluate different contacts of the process. We could verify that the $\mathrm{Ni} / \mathrm{N}$-implanted $\mathrm{SiC}$ contact was very poor (even rectifying). The contact between $\mathrm{Ti}$ and $\mathrm{B}$-implanted $\mathrm{SiC}$ was also characterized, and we found a surprisingly ohmic behaviour, with an extracted specific contact 
resistance of $\sim 3.5 \mathrm{mOhm} . \mathrm{cm}^{2}$ in the best case. This contact should be considered as a contact to $n$-type $\mathrm{SiC}$, as the implanted $\mathrm{B}$ is not supposed to be activated. This ohmic contact allowed us to measure the resistance of the SiC layers (transferred layer and epitaxial), and we could verify that, according to doping levels and thicknesses, both SiC layers exhibited non-degraded resistivities. So we believe that the low On current on the diodes is the result of a failing $\mathrm{Ni} / \mathrm{SiC}$ ohmic contact, and not due to any SiCOI substrate (and epitaxy) related problem.

Reverse bias: Reverse characteristics were measured. Very high breakdown voltage could be obtained on the devices. The best results on the SiCOI substrates with semi-insulating transferred layer, this evidencing the Resurf effect predicted by simulations. As shown in figure 9 , the $\mathrm{V}_{\mathrm{br}}$ reaches the very interesting value of $\sim 1000 \mathrm{~V}$.

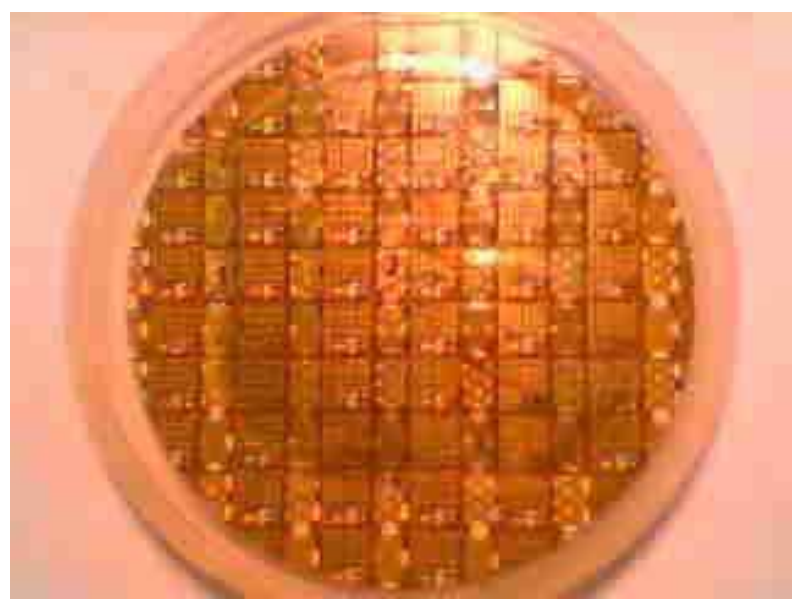

Figure 6: photograph of fully processed 2-in. SiCOI wafer with epitaxy and devices.

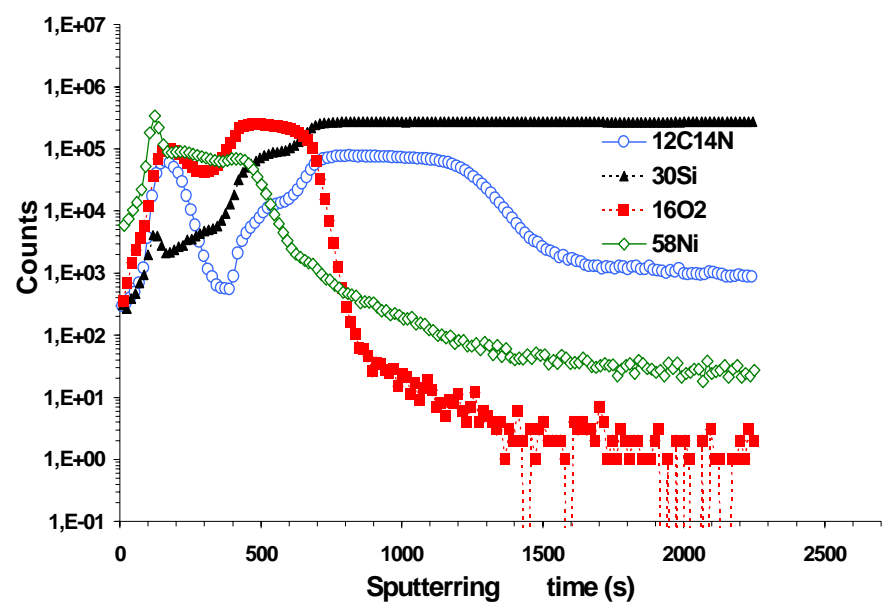

Figure 8: SIMS analysis of defective ohmic contact of Schottky diodes.

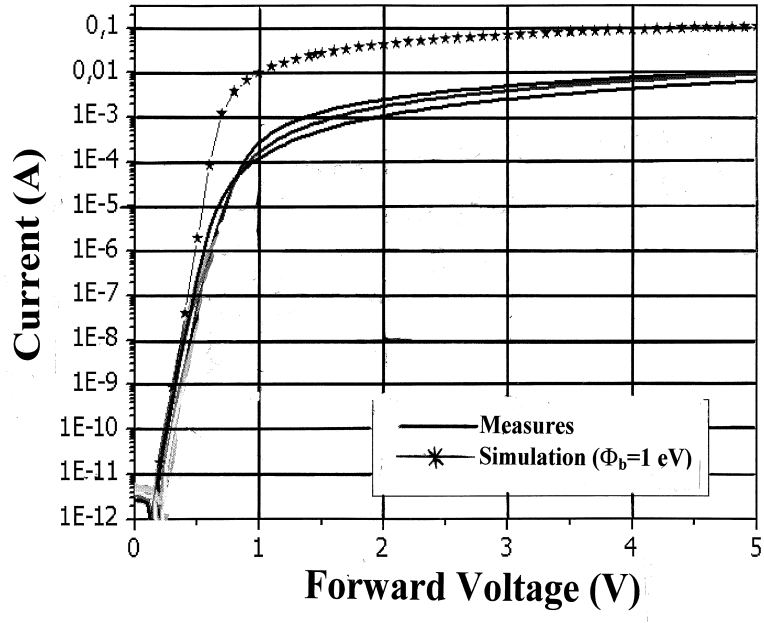

Figure 7: Forward characteristics of Schottky diode.

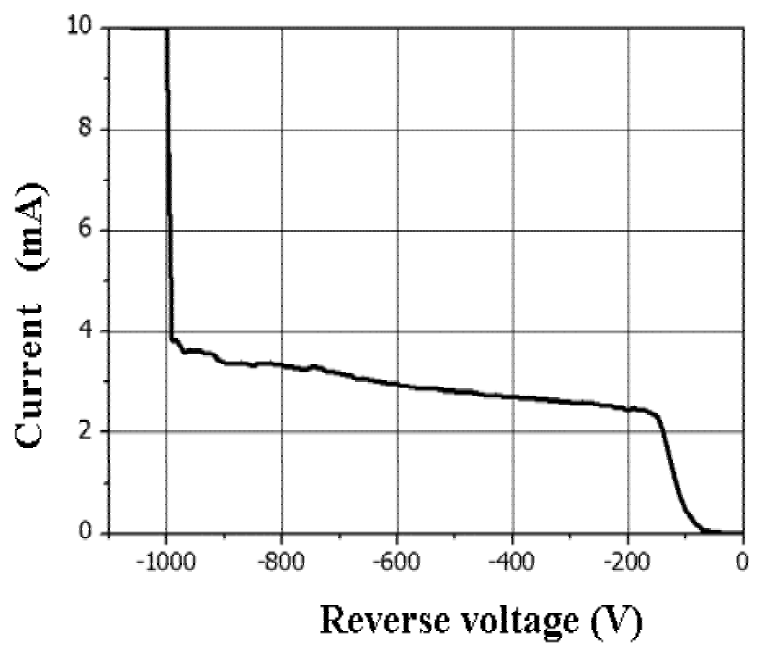

Figure 9: Reverse characteristics of Schottky diode. 
Finally, the electrical results of Schottky diodes made on SiCOI $+\mathrm{SiC}$ epitaxy can be summarized as follows: (i) the forward current is lower than expected: it was due to accidental oxygen contamination during the ohmic contact step, and is not related to SiCOI substrate and/or subsequent $\mathrm{SiC}$ epitaxy, (ii) reverse voltages are excellent, $\mathrm{V}_{\mathrm{br}}$ of $\sim 1000 \mathrm{~V}$ were obtained, (iii) the influence of doping of the transferred layer was verified.

\section{CONCLUSION}

We demonstrated the feasibility of SiC epitaxy on SiCOI substrates. Epitaxies show very good quality. As a first step for device evaluation of these new structures, we have designed and fabricated power Schottky diodes. Electrical results showed excellent reverse characteristics while the direct current was lower than expected, due to device process problem. Further work is underway to improve the ohmic contact, and demonstrate much higher current ratings, as the current limitation was independent of the SiCOI substrate and epitaxy. The possibility of SiC epitaxy on SiCOI substrate is open to any doping (type, level) and thickness, thus almost any kind of horizontal power device can be potentially realized. Moreover, the SiCOI substrate provides the unique possibility of galvanic isolation between device. Overall, we believe that this new technology is very promising for the realization of $\mathrm{SiC}$ power systems.

\section{AKNOWLEDGEMENTS}

The authors would like to greatly thank R. Madar and I. Matko from LMGP / INPG, for providing TEM characterization.

\section{REFERENCES}

1. T. Letavic and M. Simpson, US Pat. 6127703 (2000)

2. L. DiCioccio, Y. Le Tiec, F. Letertre, C. Jaussaud and M. Bruel, Electronics Letters, 32, 12 , pp. 1144-45 (1996)

3. E. Hugonnard-Bruyère, F. Letertre, L. DiCioccio, H.J. von Bardeleben, J.-L. Cantin, T. Ouisse, T. Billon and B. Guillot, Material Science Forum Vols. 338-342, 714 (2000)

4. F. Letertre, N. Daval, F. Templier, E. Bano, D. Planson, L. DiCioccio, E. Jalaguier, J.-M. Bluet, T. Billon, R. Madar and J.-P. Chante, Proc. of ECSCRM 2002, Linköping, Sweden, (2002) 5. E. Neyret, L. D. Cioccio, E. Blanquet, C. Raffy, C. Pudda, T. Billon, and J. Camassel, Material Science Forum Vols. 338-342, 1041 (2000)

6. G. Ferro, N. Planes, V. Papaioannou, D. Chaussende, Y. Monteil, Y. Stoemenos and J. Camassel, Mat. Sci. and Engineerin, B B61-62, 586 (1999)

7. M. Eickchoff, S. Zappe, A. Nielsen, G. Krötz, E. Obermeier, N. Vouroutzis and J. Stoemenos; Mat Sci. Forum, 353-356, 175 (2001)

8. see http://www.epigress.com

9. N. Daval, Etude de la faisabilité de Composants de Puissance sur substrats en Carbure de Silicium sur Isolant (SiCOI), Thesis, November $19^{\text {th }} 2002$, INSA de Lyon, (2002)

10. A. Itoh, T. Kimoto and H. Matsunami, Proc. of ISPSD 1995, Yokohama, Japan, 101 (1995). 11. R. Rupp, M. Treu, A. Mauder, E. Griebl, W. Werner, W. Bartsch and D. Stephani, Mat. Sci. Forum, 338-342, 1167 (2000) 